\title{
sciendo
}

DOI: $10.2478 / f v-2019-0040$

FOLIA VETERINARIA, 63, 4: 70-77, 2019

\section{COMBINATION OF BETA GLUCAN, HONEY AND CHLORHEXIDINE IN THE WOUND MANAGEMENT IN A CAT A CASE REPORT}

\author{
Michál’ová, A. ${ }^{1}$, Michál', M. $^{2}$, Fialkovičová, M. ${ }^{1}$ \\ ${ }^{1}$ University of Veterinary Medicine and Pharmacy in Košice, Komenského 73, 04181 Košice \\ ${ }^{2}$ Animed s.r.o., Borovianska 76, 96001 Zvolen \\ Slovakia \\ maria.fialkovicova@uvlf.sk
}

\begin{abstract}
Wound management is one of the oldest and one of the most frequent therapeutic activities in medicine. Over the centuries there has been described and tested many therapeutic substances for the treatment of wounds with various effects. Due to the discovery of antibiotics, a wound management regime used to be limited only to a local application. Over years, it has been shown, that comprehensive therapy which uses only antibacterial preparations, also may contain some negative points (resistance of aggressive pathogens, toxicity, allergic reactions, etc.). According to studies, the best solution to this problem is a local application, using preparations that ensure the sterility of the affected parts of the skin, and the utilization of agents that are able to accelerate the granulation and lead to the healing process of the wound. Products that contain beta glucan combined with a gentle but effective antiseptics (chlorhexidine digluconate), or natural substance with antiseptic and also nourishment effect (honey), are very beneficial in wound management. A good penetration of active agents, the
\end{abstract}

ability to moisturize defective tissues, and to make a protective film that hinder the intersection of impurities and decrease secondary contamination, are the benefits of a gel formulation, that is the most appropriate external form of application in veterinary practice that can improve and accelerate a successful healing process of wounds in animals.

Key words: beta glucan; chlorhexidine digluconate; honey; wounds

\section{INTRODUCTION}

Beta glucan

Beta glucans belongs to prominent immunomodulators and activators of white blood cells, especially macrophages which are able to significantly accelerate the wound healing process and its positive effect is manifested in all phases of healing. Glucans are long-chain polysaccharides. They are isolated from Saccharomyces and have an ability to improve and promote immunity which has become compro- 
mised mainly in the case of long term diseases [2]. In addition to the immuno-stimulatory effect, they also have antiinfective activity and positive effects on cancer and increase the body's resistance to chemical and carcinogenic agents $[11,28,31]$. Beta glucan (BG) is mainly present in yeast and other fungal organisms, and it is composed of glucose polymers that have a positive effect on: the growth of bacterial probiotic strains in the intestinal tract, improve the blood lipid profile, and stimulate cellular immunity by the limiting of various secondary immuno-deficiencies developments due to the limitation of immune cell activation [25, 37]. The application of beta-(1,3/1,6)-D-glucan activates macrophages which are able to remove disintegrated parts of cells, foreign particles and pathogenic microorganisms, that significantly stimulate the natural immune response [34]. The main mechanisms of action are: binding to the $B G$ receptor which is found on the surface of macrophages, neutrophils, monocytes and natural killer (NK) cells, and stimulation of the natural immune response [30]. These activated macrophages have: the ability to release large amounts of active substances (lysozyme, complement components, etc.), have a bactericidal effect, involve immunomodulatory processes through the secretion of cytokines (granulocyte macrophage colony stimulating factor (GM$\mathrm{CSF}$ ), stimulates the growth of hematopoietic cells, interleukin-1 that is a major cytokine for T lymphocytes activation), participates in antigen presentation processes, and interleukin-2 production. Interleukin-12 promotes NK-cell activity and is involved in the anti-proliferative, immunoregulatory and antiviral action of macrophages $[4,5,20$, 29]. Other mechanisms of action of BG are including its binding ability to the complement receptor, which acts as an adhesive molecule. In addition, the effects of some $\beta$-glucans have also been anti-infective and have antibacterial activity against a broad spectrum of Gram-negative and Gram-positive bacteria [4]. Beta-(1, 3/1,6)-D-glucan has very beneficial effects in the healing of ulcerations and wounds $[17,18]$. Its binding areas are also found on dermal fibroblasts, keratinocytes and have the ability to functionally modulate these cells by stimulation of macrophages that prevent many disorders. The results of studies and the practical use of $B G$, confirm an increased protective effect against bacterial, viral, parasitic and fungal infections in all animal categories [30]. Its use in practice generally increases the activity of the immune response, alleviating clinical signs of disease reducing the process of healing and secondary infections, improving the efficacy of causal therapy and the prognosis of healing. Beta glucan also has an adjuvant effect in enhancing the effectiveness of therapy with other drugs such as antibiotics (ATB) and increase the effectiveness of the immune response after vaccination [9], and it has also been safe and non-toxic for animals [35].

The effects of beta-(1,3/1,6)-D-glucan include:

- Increase immunity system against infections (viral, bacterial, parasitic and fungal),

- Adjuvant effect -- promotes the effectiveness of vaccination and antibiotic therapy,

- Stimulation of other cells of the immune system (cytotoxic T-lymphocytes and NK-cells) and subsequent anticancer action,

- Support of regeneration and repair processes through fibroblast and keratinocyte activation promoting wound healing and reducing ulceration,

- Shortens recovery period,

- Radio-protective effect due to inactivation of free radicals,

- Promotion of haematopoesis and bone marrow activity.

\section{Honey}

Honey is a saturated solution of sugars, composed mainly of fructose, glucose, water, minerals, vitamins, enzymes, free amino acids, polyphenols, and around 0.17-1.17\% of organic acids. Pure bee honey has antibacterial effects and the ability to moisturize the wound. Its high viscosity helps to create a protective barrier that protects against infection. Its immunomodulation and antimicrobial activity is due to the enzymatic production of hydrogen peroxide, low $\mathrm{pH}$ and high osmolality that can sufficiently prevent the growth of microorganisms $[12,14,27,38]$. The antimicrobial activity of honey has been described "in vivo" conditions, by the direct application of honey to the wound, which in 3-6 days was marked by its ability to inhibit microbial growth, because sugar molecules build up water molecules and cause bacteria to have insufficient water for their growth [1]. Honey effectively delivers hydrogen peroxide which is slowly released, because the enzyme that produces it becomes active only when honey is diluted and continues to its stable production during at least 24 hours. If honey is diluted with a buffer in the same proportion, the hydrogen peroxide concentration collected over 1 hour is about 1000 times less, than the concentration in the solu- 
tion of $3 \%$ hydrogen peroxide, which is commonly used as an antiseptic. Honey also contains a relatively large amount of antioxidants that protect wound tissues from oxygen radicals that could be produced by hydrogen peroxide [7].

\section{Chlorhexidine digluconate}

Chlorhexidine is a broad-spectrum biocidal antiseptic that is effective against fungi, gram-positive and gram-negative bacteria. It inhibits bacterial growth and also acts as a bactericidal agent. It can kill nearly $100 \%$ of the grampositive and gram-negative bacteria within 30 seconds. This effective skin antiseptic is beneficial in the prevention of various infections, injuries, and surgical procedures. Microorganisms that colonize the skin are found on the top of skin surface, in hair follicles and also in deeper layers of the skin. Many antimicrobial agents are not able to penetrate into the deeper layers of the skin and hair follicles where there could be a presence of coagulase-negative staphylococci $[8,16,26]$. Chlorhexidine binds to proteins, releases slowly, and has longer lasting effects against microorganisms [38]. Its antimicrobial activity is maintained on the skin for at least 48 hours [10]. The efficacy has not been affected by the presence of body fluids such as blood [19]. Also it is able to inhibit the adherence of microorganisms on the surface, and blocks the creation and growth of the biofilm [23].

\section{MATERIALS AND METHODS}

According to studies focused on the use of beta glucan, pure bee honey and chlorhexidine digluconate individually in the wound management and the positive and beneficial effects of each substance, we created a gel, where all three substances together were represented and we observed in post-traumatic wound in the cat, if the treatment and time duration of the complete healing would be effective, faster and without any side effects. The gel was applied once daily throughout the process of complete wound recovery. We administered also oral suspension of beta 1,3/1,6 D-glucan in combination with ascorbic acid and zinc (Imulergan Compositum susp. a.u.v.) that was administered during all treatment periods.

\section{CASE PRESENTATION}

\section{Anamnesis}

A nine-year-old cat "Lisa" had a free movement between the outside and inside of her home. During her stay outside her owners assumed that the cat was caught in a knocker and hurt. Her skin on the right front limb was totally peeled off and she was pulling the distal part of her front limb behind her. The cat was immediately brought to a veterinary clinic where she was treated. The torn joint was fixed and the remaining skin was sewed. Antibiotics were immediately administrated to the cat (Cefalexin a. u.v. inj., $50 \mathrm{ml}$ ) for seven days, and Metacam $0.5 \mathrm{mg} \cdot \mathrm{ml}^{-1}$ given once daily to reduce the pain and to relieve the inflammation. Later it was necessary to perform a plastic skin surgery to the patient. We provided an artificial skin replacement but the wound was damp and drippy.

\section{Diagnosis}

Post trauma necrosis of the skin and subcutaneous structures.

\section{Treatment}

After plastic skin surgery, the wound healing was very difficult. The process of healing was protracted, because the wound had to be covered and protected. According to the status of the animal, we began with an oral administration of $2 \mathrm{ml}$ of beta glucan oral suspension (Imulergan Compositum susp. a.u.v.), that consisted of beta 1,3/1,6 D-glucan, and ascorbic acid and zinc, which potentiate the effect of BG. This peroral application of beta glucan was served throughout the treatment period. Also given was a topical application of gel containing beta glucan, chlorhexidine digluconate and pure bee honey to the wound once daily (Fig. 1), where a significant improvement after every application was observed. The enhancement was recorded after five (Fig. 2), ten (Fig. 3), and fifteen (Fig. 4) days of gel application. After 2 weeks of treatment we examined the blood for all relevant blood parameters. The blood test parameters were in the normal range, so additional administration of antibiotics was unnecessary. The wound did not have to be covered anymore after 4 weeks of treatment. The topical application of gel continued for an additional two weeks and after 6 weeks of therapy we terminated the topical application of beta glucan containing gel because the wound was healed. Treatment was maintained 


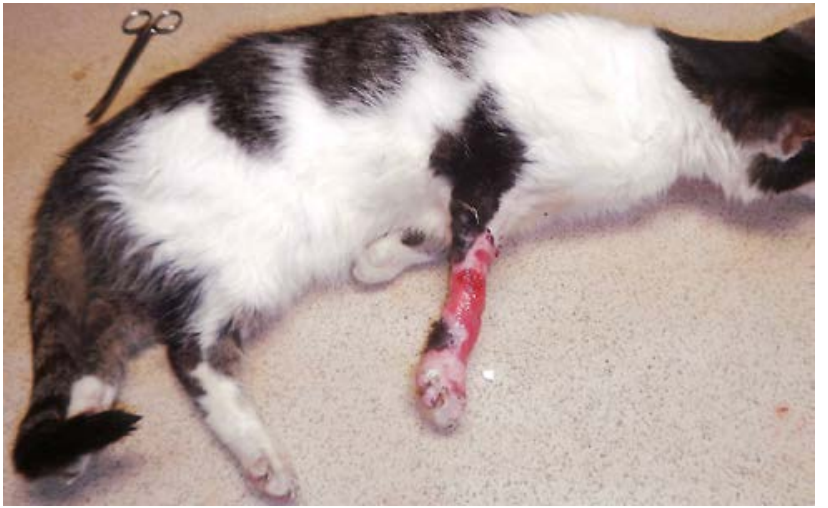

Fig. 1. First day of the product application

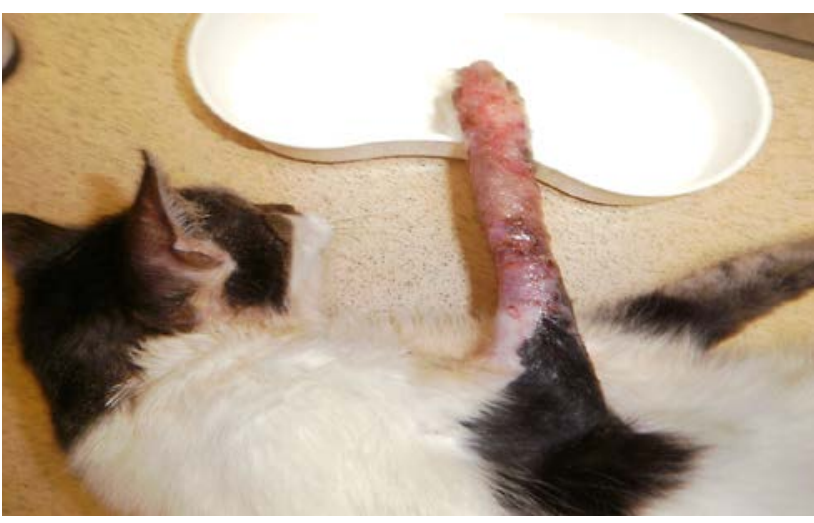

Fig. 3. Ten days after first application

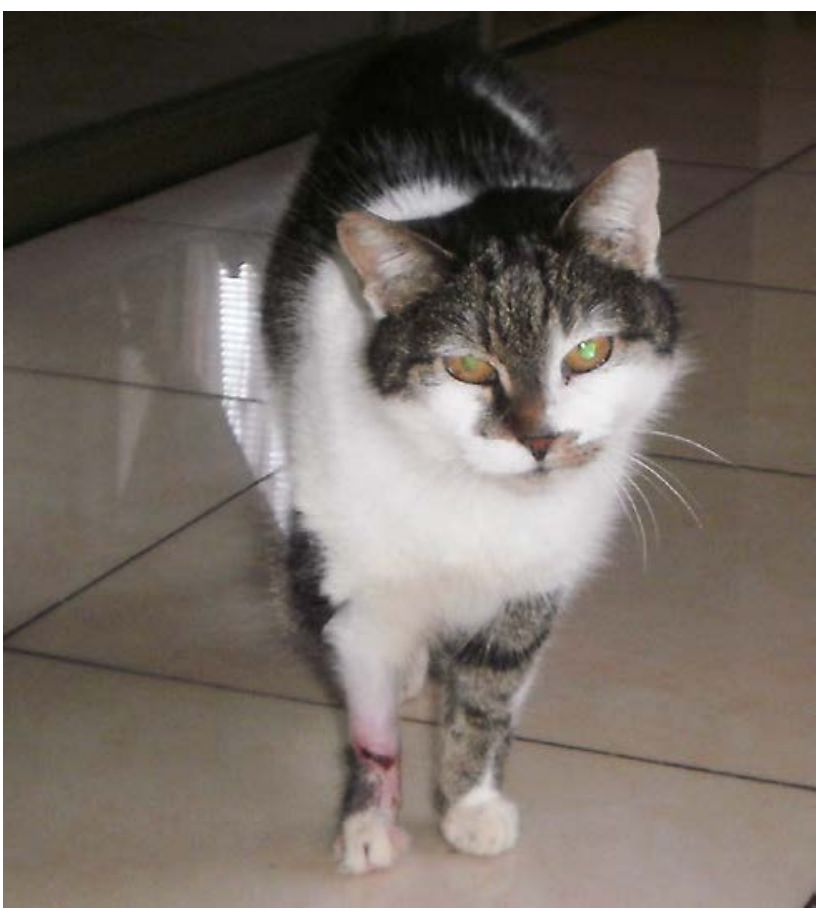

Fig. 5. After 35 days of product application

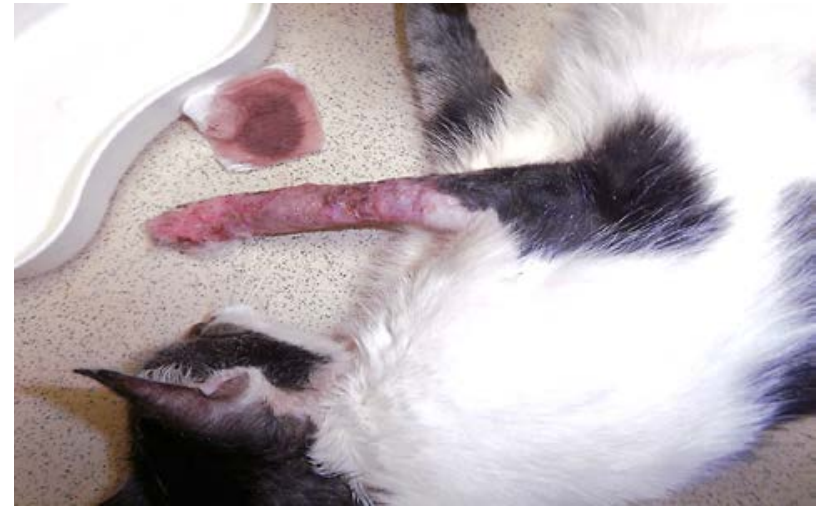

Fig. 2. Five days after first application

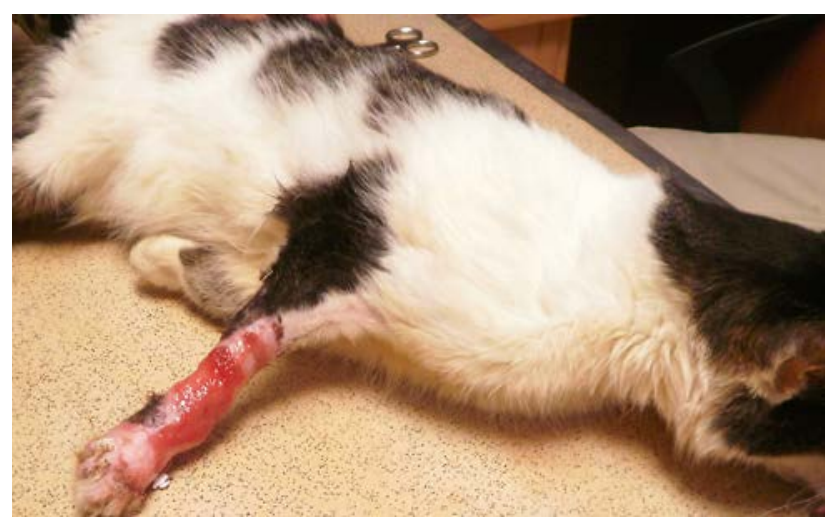

Fig. 4. Fifteen days after first application

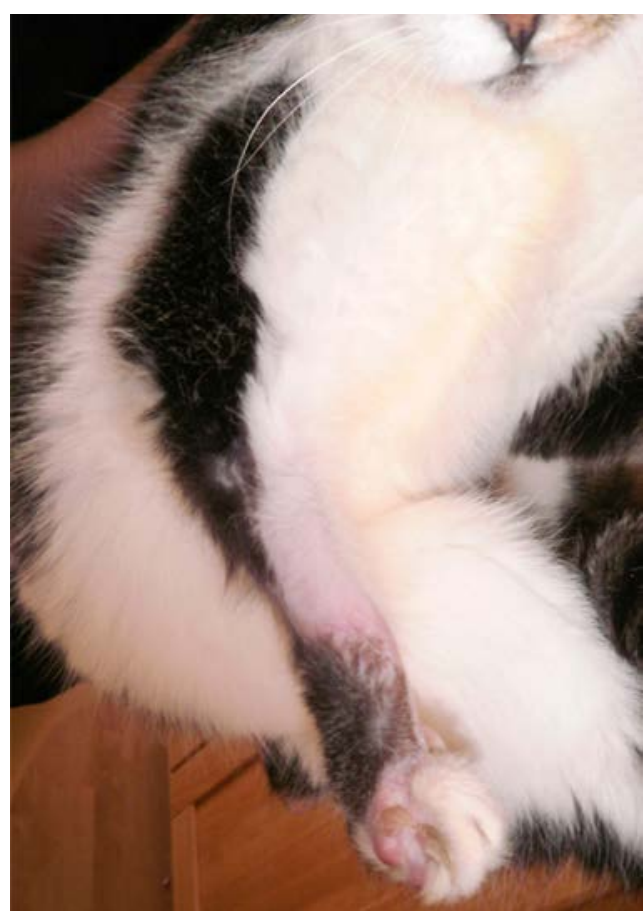

Fig. 6. The cat after 10 weeks of therapy 
only with the oral administration of beta glucan during an additional 4 weeks. After 35 days of treatment (Fig. 5) the condition of the animal was significantly improved, and after 10 weeks of therapy complete healing occurred and her hair began to grow again (Fig. 6).

\section{DISCUSSION}

The topical use of beta glucan, pure honey or chlorhexidine digluconate in wound treatments have been proven in a number of various studies around the world independently [1, 10,39]. All results of these studies concentrated on the usage of BG and confirmed its ability to stimulate cell-mediated and partial humoral immunity, mechanisms of non-specific immunity and haematopoiesis [36]. The oral administration of beta glucan demonstrated positive effects on the altered immune parameters. Their improvement was correlated with the clinical picture of the investigated dogs, which in the case of pyoderma and Malassezia showed skin improvement. The positive effects of BG have also been demonstrated in the case of immunosuppressed parvovirus dogs after their vaccination. After the first day following an administration of BG, there was observed: an increased phagocytic activity, the stimulation index increased significantly, and the antibody titre was higher in the observed group of patients [9]. In addition to antibiotic therapy, BG was administered to patients with impaired health. There was a significant clinical improvement after BG was applied to patients with atopic allergy, folliculitis and non-specific upper respiratory tract inflammation. All clinical observation studies have confirmed that BG preparations had no adverse side effects and were well tolerated $[12,14]$.

Z y k o va et al. [39] studied a topical use of BG in patients with diabetic foot and leg ulcers and have confirmed that in skin infections associated with various degrees of immunosuppression, it is necessary to apply an immunomodulatory therapy. The immunostimulatory effect of BG on the functional activity of lymphocytes and phagocytes were also evaluated in skin diseases in dogs affected by dermatomycosis, demodicosis and pyoderma [22]. The clinical study of the topical application of BG under laboratory conditions was conducted by the Biotec company and was focused on patients to whom the formulation was applied twice daily for coverage of the primary wound in diabetic ulcers, pressure ulcers, leg ulcers, postoperative wounds, first and second degree burns, abrasions and skin wounds. The formulation was administrated directly to the open wounds, and every other day to wounds covered with a primary dressing until complete wound healing during 12 weeks [39]. Its application with antibiotic therapies was also very beneficial and its effect reduced any possibility of the formation of mutated bacterial strains resistant to ATB. Glucan is able to enhance an intensity of immune response and accelerates an onset of specific antibody production upon vaccination. By local application, it can positively influence wound healing, optimize surgical wound regeneration and contribute to the elimination of secondary infections after surgery [29]. Beta glucan application increases the cell immunity reaction and capacity of immune system, is safe, can be used as a supportive therapy, and can combine with other remedies and chemical substances that act synergistically. Beta glucan in prophylaxis allows the body to activate defence mechanisms which helps to relieve stress and prevent diseases [6]. It has the same mechanism of action in humans and other animals, so it can be used in pets (dogs, cats, parrots), cattle, pigs, horses, farmed freshwater and sea fish as well as other sea animals (shrimps, lobsters, crabs). Nowadays, glucan is regarded as useful due to its beneficial effects to the organism; a part of feed and nutritional supplements for improving the health of all types of domestic animals and fish [31].

Honey has been used in the treatment of many types of wounds, including burns, traumatic, surgical, necrotizing or ulcerative injuries. In the case of burns, the treatment with honey has been more effective than other products. Some studies have also shown that the effects of honey are as effective in the treatment of burns as silver sulfadiazine. When comparing honey and silver sulfadiazine, it was found that in acute, partial burns, the application of honey provided an early reduction of acute inflammatory changes, better infection control, faster healing process, decreased irritation and exudation, pain relief, reduced hypertrophic scar and post burn contractures $[32,33]$. Studies also confirm the total inhibition of MRSA (Methicillin-Resistant Staphylococcus aureus), full inhibition of 58 coagulase-positive Staphylococcus aureus strains, isolated from infected wounds and complete inhibition from 20 Pseudomonas strains, also isolated from infected wounds [12, 14, 27, 38]. In a comparative study between honey and hydrogen peroxide, as a single antiseptic in wound therapy, the single 
hydrogen peroxide had been less beneficial, although its presence in honey was used in a different way. This possibility is due to a sufficiently high concentration of hydrogen peroxide that cause the breakdown of proteins and cells in the tissues by increasing oxygen radicals, which limited the concentration of hydrogen peroxide as an antiseptic [24].

Compared to other antimicrobial agents, chlorhexidine has been antibacterial in the case of protozoa and bacterial spores covered by the virus $[3,21]$. According to several studies it was confirmed that chlorhexidine has had an ability to inhibit an adherence of microorganisms to the surface and is able to prevent formation and growth of a biofilm [36].

\section{CONCLUSIONS}

Beta glucan has immunomodulatory and granulation effects, chlorhexidine is an effective disinfectant and antiseptic even in low concentrations, and honey nourishes the wound and creates a hyperosmotic environment that prevents bacterial growth. According to other studies and our experience we can confirm that:

1. Topical administration of beta glucan in a complex formulation in combination with other agents that potentiate its effect, makes the therapy easier and more effective (instead of a multi-stage wound treatment application the only one preparation).

2. A disinfectant such as chlorhexidine digluconate, and pure bee honey act synergistically with beta glucan and complement its effect.

3. Gel formulation seems to be the most suitable vehicle for animals if compared to other forms:

4. Solutions: short effect, worse penetration,

5. ointment, cream: worse application possibilities,

6. powder: dries a wound, limited indication.

7. Beta glucan in this form has been shown to be safe and hypoallergenic, since we used it in the formulation BG isolated from Pleurotus ostreatus (Oyster mushroom) so there were no reactions or side effects observed during its administration.

8. The formulation has also hydration effects so there was no irritation or any secondary complications caused by pruritus.

9. The healing process was shortened by approximately $30 \%$.

The objective of this case report was to remind others, that the use of beta glucan, which has often been studied and monitored, has been a very beneficial, safe and inexpensive substance with a strong immunomodulatory and adjuvant effect providing regeneration and repair processes. BG in combination with other components (chlorhexidine digluconate and pure bee honey) leads to significant shortening of the healing process without any application risks and unnecessary pharmacotherapeutic costs. Of course there is a need for further studies to confirm exactly the time duration and effectiveness of the treating process in other animals and case studies.

\section{REFERENCES}

1. Al-Waili, N. S., Salom, K., Butler, G., Al Ghamdi, A. A., 2011: Honey and microbial infections: a review supporting the use of honey for microbial control. J. Med. Food., 14, 10, 1079-96. DOI: 10.1089/jmf.2010.0161.

2. Bohn, J. A., BeMiller, J. N., 1995: ( $1 \rightarrow 3)$-b-d-Glucans as biological response modifiers: A review of structure-functional activity relationships. Carbohydr. Polym., 28, 3-14. DOI: 10. 1016/0144-8617(95)00076-3.

3. Boyce, J. M., Pittet, D., 2013: Guideline for hand hygiene in health-care settings: recommendations of the Healthcare Infection Control Practices Advisory Committee and the HICPAC/SHEA/APIC/IDSA Hand Hygiene Task Force. Infection Control and Hospital Epidemiology, 23, 12 Suppl., S340. DOI: $10.1086 / 503164$.

4. Chamidah, A., Hardoko, Prihanto, A. A., 2017: American Institute of Physics Antibacterial activities of $\beta$-glucan (laminaran) against gram-negative and gram-positive bacteria. AIP Conf. Proc., 1844, 020011. DOI: 10.1063/1.4983422.

5. Chinnu. K., Muthukumaran, M., Mukund, S., Sivasubramanian, V., 2014: Antimicrobial and antifungal activity of isolated betaglucan from chroococcus turgidus. Indian J. Pharm. Sci. Res., 4, 217-220.

6. Chovancová, A., Šturdík, E., 2005: The influence of betaglucans on the immune system of man (In Czech). Nova Biotechnologica, V-1. 105-121.

7. Frankel, S., Robinson, G. E., Berenbaum, M. R., 1998: Antioxidant capacity and correlated characteristics of 14 unifloral honeys. J. Apic. Res., 37, 27-31. DOI: 10.1080/00218839.1998. 11100951.

8. Guardabassi, L., Houser, G. A., Frank, L. A., Papich, M. G., 2008: Guidelines for Antimicrobial Use in Dogs and Cats. 
Guide to Antimicrobial Use in Animals. Oxford: Blackwell Publ., 182-206. ISBN: 978-1-405-15079-8.

9. Haladová, E., Mojžišová, J., Smrčo, P., Ondrejková, A., Vojtek, B., Prokeš, M., Petrovová, E., 2011: Immunomodulatory effect of glucan on specific and nonspecific immunity after vaccination in puppies. Acta Vet. Hung., 59, 1, 77-86. 14. DOI: 10.1556/AVet.59.2011.1.7.

10. Hibbard, J., 2005: Analysis comparing the antimicrobial activity and safety of current antiseptics: a review. J. Infus. Nurs., 28, 3, 194-207. DOI: 10.1097/00129804-200505000-00008.

11. Jantova, S., Bakos, D., Birosova, L., Matejov, P., 2015: Biological properties of a novel coladerm-beta glucan membrane. In vitro assessment using human fibroblasts. Biomedical Papers of Medical Faculty of the University Palacky, Olomouc, 159, 67-76. DOI: 10.5507/bp.2012.115.

12. Jull, A. B., Walker, N., Deshpande, S., 2013: Honey as a topical treatment for wounds. Cochrane Database Syst. Rev., 2, CD005083. DOI: 10.1002/14651858.CD005083.pub3.

13. Karaaslan, O., Kankaya, Y., Sungur, N., Kocer, U., Cuzdan, S., Sahin, B., Uysal, A., 2012: Case series of topical and orally administered $\beta$-glucan for the treatment of diabetic wounds: Clinical study. J. Cutan. Med. Surg., 16, 180-186. DOI: 10. 1177/120347541201600308.

14. Khan, F. R., Ul Abadin, Z., Rauf, N., 2007: Honey: Nutritional and medicinal value. Int. J. Clin. Pract., 61, 10, 17051707. DOI: 10.1111/j.1742-1241.2007.01417.x.

15. King, B., Barrett, S., Cutting, K. F., 2017: Clinical evaluation of a bioactive beta-glucan gel in the treatment of 'hardto-heal' wounds. J. Wound Care, 26, 58-63. DOI: 10.12968/ jowc.2017.26.2.58.

16. Krautheim, A. B., Jermann, T. H., Bircher, A. J., 2004: Chlorhexidine anaphylaxis: case report and review of the literature. Contact Dermat., 50, 113-116. DOI: 10.1111/j.01051873.2004.00308.x.

17. Kwon, A. H., Qiu, Z., Hashimoto, M., Yamamoto, K., Kimura, T., 2009: Effects of medicinal mushroom (Sparassis crispa) on wound healing in streptozotocin-induced diabetic rats. Am. J. Surg., 197, 4, 503-509. DOI: 10.1016/j.amjsurg. 2007.11.021.

18. Lee, J. W., Kwon, Y. S., Jang, K. H., 2012: Effect of $\beta$-glucan originated from Aureobasidium on infected dermal wound healing of the normal nude mouse. J. Vet. Clin., 29, 464-469. DOI: 10.12729/jbr.2015.16.4.140.

19. Lim, K. S., Kam, P. C., 2008: Chlorhexidine - pharmacology and clinical applications. Anaesth. Intensive Care, 36, 4, 502-512. DOI: 10.1177/0310057X0803600404.
20. Lundahl, M. L. E., Scanlan, E. M., Lavelle, E. C., 2017: Therapeutic potential of carbohydrates as regulators of macrophage activation. Biochem. Pharmacol., 146, 23-41. DOI: 10. 1016/j.bcp.2017.09.003.

21. Mcdonell, G. E., Russel, A. D., 1999: Antiseptics and disinfectants: activity, action and resistance. Clin. Microbiol. Rev., 12, 1, 147-179. DOI: 10.1128/CMR.12.1.147.

22. Medeiros, S. D., Cordeiro, S. L., Cavalcanti, J. E., Melchuna, K. M., Lima, A. M., Filho, I. A., et al., 2012: Effects of purified Saccharomyces cerevisiae $(1 \rightarrow 3)-\beta$-glucan on venous ulcer healing. Int. J. Mol. Sci., 13, 8142-8158. DOI: 10.3390/ ijms13078142.

23. Mohammadi, Z., Abbott, P. V., 2009: The properties and applications of chlorhexidine in endodontics. Int. Endodont. J., 42, 4, 288-302. DOI: 10.1111/j.1365-2591.2008.01540.x.

24. Molan, P. C., 1992: The antibacterial activity of honey. 2: Variation in the potency of the antibacterial activity. Bee World, 73, 2, 59-76.

25. Nicolasi, R., Bell, S. J., Bistrian, B. R., Greenberg, I., Forse, R. A., Blackburn, G. L., 1999: Plasma lipid changes after supplementation with beta-glucan fiber from yeast. Am. J. Clin. Nutr., 70, 208-212.

26. Sayed, H. A., Gamal, M. M., Mohamed, A. I., Gamal, A. S., Mounir, M.S. B., 2017: Novel chlorhexidine dermal patches, preparation characterization and antimicrobial evaluation. Polymer Bulletin, 74, 10, 3995-4007. DOI:10.1007/ s00289-017-1935-0.

27. Sherlock, O., Dolan, A., Athman, R., Power, A., Gethin, G., Cowman, S., et al., 2010: Comparison of the antimicrobial activity of Ulmo honey from Chile and Manuka honey against methicillin-resistant Staphylococcus aureus, Escherichia coli and Pseudomonas aeruginosa. BMC Complement Altern. Med., 10, 1, 1-5. DOI: 10.1186/1472-6882-10-47.

28. Sherwood, E. R., Williams, D. L., McNamee, R. B., Jones, E. L., Browder, I. W., DiLuzio, N. R., 1987: In vitro tumoricidal activity of resting and glucan-activated Kupffer cells. J. Leukoc. Biol., 42, 69-75. DOI: 10.1002/ jlb.42.1.69.

29. Shin, M. S., Lee, S., Lee, K. Y., Lee, H. G., 2005: Structural and biological characterization of aminated-derivatized oat beta-glucan. J. Agric. Food Chem., 53, 5554-5558. DOI: 10. 1021/jf050273j.

30. Singer, A. J., Clark, R. A., 1999: Cutaneous wound healing. N. E. J. Med., 341, 738-746. DOI: 10.1056/NEJM1999090 23411006.

31. Stier, H., Ebbeskotte, V., Gruenwald, J., 2014: Immune- 
modulatory effects of dietary Yeast Beta-1,3/1,6-D-glucan. Nutr. J., 13, 1-9. DOI:10.1186/1475-2891-13-38.

32. Subrahmanyam, M., 1991: Topical application of honey in treatment of burns. Br. J. Surg., 78, 4, 497-498.

33. Subrahmanyam, M., 1998: Prospective randomised clinical and histological study of superficial burn wound healing with honey and silver sulfadiazine. Burns, 24, 2, 157-161. DOI:10.1016/s0305-4179(97)00113-7.

34. Sun, L., Zhao, Y., 2007: The biological role of dectin-1 in immune response. Int. Rev. Immunol., 26, 349-364. DOI: 10.1080/08830180701690793.

35. Vannucci, L., Krizan, J., Sima, P., Stakheev, D., Caja, F., Rajsiglova, L., et. al., 2013: Immunostimulatory properties and antitumor activities of glucans. Intern. J. Oncol., 43, 2, 357-364. DOI:10.3892/ijo.2013.1974.

36. Vetvicka, V., Novak, M., 2011: Biological action of $\beta$-glucan. In Vetvicka, V., Novak, M. (Ed.): Biology and Chemistry of Beta Glucan, Vol. 1: Beta Glucans-Mechanisms of Action. Bentham Press, 10-25. DOI: 10.1007/s13596-011-0024-4.
37. Wang, Q., Sheng, X., Shi, A., Hu, H., Yang, Y., Liu, L., et al., 2017: $\beta$-glucans: Relationships between modification, conformation and functional activities. Molecules, 22, 257. DOI: 10. 3390/molecules22020257.

38. Zohdi, R. M., Zakaria, Z. A. B., Yusof, N., Mustapha, N. M., Abdullah, M. N. H., 2012: Honey-based hydrogel as burn wound dressing. Evid. Based Complement. Alternat. Med., 2012, 1-7. DOI: 10.1155/2012/843025.

39. Zykova, S. N., Balandina, K. A., Vorokhobina, N. V., Kuznetsova, A. V., Engstad, R., Zykova, T. A., 2014: Macrophage stimulating agent soluble yeast $\beta-1,3 / 1,6$-glucan as a topical treatment of diabetic foot and leg ulcers: A randomized, double blind, placebo-controlled phase II study. J. Diabetes Investig., 5, 392-399. DOI: 10.1111/jdi.12165.

Received September 3, 2019

Accepted October 19, 2019 